\title{
Optimized biological tools: ultrastructure of rodent and bat teeth compared to human teeth
}

\author{
Alwina Luebke PhD \\ Inorganic Chemistry and Center for Nanointegration Duisburg-Essen, \\ University of Duisburg-Essen, Essen, Germany \\ Kateryna Loza PhD \\ Inorganic Chemistry and Center for Nanointegration Duisburg-Essen, \\ University of Duisburg-Essen, Essen, Germany \\ Oleg Prymak PhD \\ Inorganic Chemistry and Center for Nanointegration Duisburg-Essen, \\ University of Duisburg-Essen, Essen, Germany
}

\author{
Philip Dammann PhD \\ Department of General Zoology, University of Duisburg-Essen, \\ Essen, Germany; Central Animal Laboratory, University Clinics Essen, \\ Essen, Germany \\ Helge Otto Fabritius PhD \\ Microstructure Physics and Alloy Design, Max-Planck-Institut für \\ Eisenforschung, Düsseldorf, Germany \\ Matthias Epple PhD \\ Inorganic Chemistry and Center for Nanointegration Duisburg-Essen, \\ University of Duisburg-Essen, Essen, Germany (corresponding author: \\ matthias.epple@uni-due.de) (Orcid:0000-0002-1641-7068)
}

Enamel, the outer layer of the teeth of vertebrates, is the hardest tissue in their body. In general, enamel is highly mineralized and has a special ultrastructure that directs the mechanical properties of teeth and thereby their biological functions. However, there are differences between the enamel ultrastructures of different species. The authors compare the teeth of the Eurasian beaver Castor fiber, the African mole rat Fukomys kafuensis and the common pipistrelle bat Pipistrellus pipistrellus by high-resolution analytical methods, including scanning electron microscopy, quantitative wavelength-dispersive X-ray spectroscopy and X-ray powder diffraction. The enamel of all animals consists of long, thin and differently oriented calcium phosphate crystallites (length > 1 um; thickness about $50 \mathrm{~nm}$ ). On the outer surface of the beaver tooth, a thin layer of brown iron oxide was found. The diameter of the dentin tubuli was between 1 and $3 \mu \mathrm{m}$ for all species - that is, comparable to that for human teeth.

\section{Notation}

$R_{\mathrm{wp}} \quad$ weighted profile $R$-factor

\section{Introduction}

Teeth represent the hardest tissue in vertebrates. As in several different hard tissues in nature, their increased hardness arises not only from their material alone, but also from their delicate ultrastructure. $^{1-3}$ In most taxa, including mammals, fish and sauropsids, bone and teeth consist of carbonated hydroxyapatite $\left(\mathrm{Ca}_{5}\right.$ $\left.\left(\mathrm{PO}_{4}\right)_{3} \mathrm{OH}\right)$, the mineral dahllite. ${ }^{4-7}$ In contrast to dentin, which contains $\sim 30 \mathrm{wt} \%$ organic matrix, tooth enamel is much more highly mineralized and contains only $\sim 1 \mathrm{wt} \%$ organic matrix. ${ }^{5,8,9}$ In mammalian tooth enamel, the mineral phase hydroxyapatite forms needle-like crystallites. Over the course of evolution, these crystallites have developed into distinct types of arrangements in different taxa that range from relatively uniform parallel orientation (parallel crystallite enamel) over the formation of columnar structures, microunits and compound units of various shapes to regular bundles termed 'enamel prisms'. ${ }^{10}$ The crystallite architecture in the enamel is denoted by the German word 'schmelzmuster', which is also used in English. ${ }^{11}$ The ultrastructure is closely related to the function of the tooth - that is, the diet of the respective organisms - which is supported by the fact that similar schmelzmusters have appeared convergently across different taxa during evolution. ${ }^{10}$

In general, enamel crystallites originate at the dentin-enamel junction (DEJ) where the forming enameloblasts are located, and their orientation is more or less perpendicular to the tooth surface. $^{10,12-16}$ The hierarchical structure and the resulting mechanical properties of biological hard tissues, including teeth, serve as inspiration to create biomimetic and bioinspired materials. ${ }^{17,18}$ Here, the authors describe the structural organization and mineral composition of the teeth of two rodents (Castor fiber and Fukomys kafuensis) and one bat (Pipistrellus pipistrellus) in comparison to those of a human (deciduous) tooth.

\section{Materials and methods}

Teeth of $C$. fiber were provided by O. Zink, Museum der Westlausitz, Kamenz, Germany. From all skulls, incisivi had been excavated from the jaw. Teeth (incisive) of F. kafuensis were provided by Professor Dr H. Burda/Dr P. Dammann, Department of General Zoology, University of Duisburg-Essen. Teeth of $P$. pipistrellus were provided by Professor Dr. M. Epple and taxonomically determined by Dr. H. Fabritius. The deciduous human tooth was obtained from a 12-year-old male. The samples were separated into the individual tissues (dentin and enamel), as described later. Scanning electron microscopy (SEM) was used to visualize the internal structure of enamel, dentin and the DEJ.

The teeth were immersed in liquid nitrogen for $2 \mathrm{~min}$ and mechanically broken into two pieces for axial freeze fracture. Secondary electron microscopy and qualitative energy-dispersive $\mathrm{X}$-ray spectroscopy (EDX) were both carried out with an ESEM 


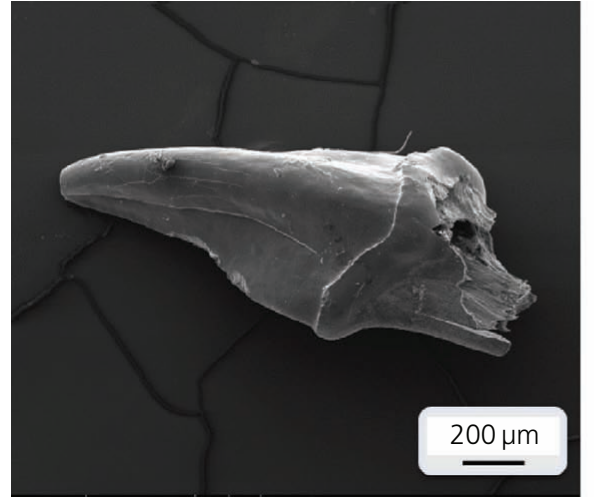

(a)

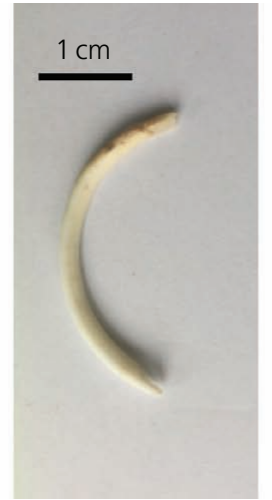

(b)

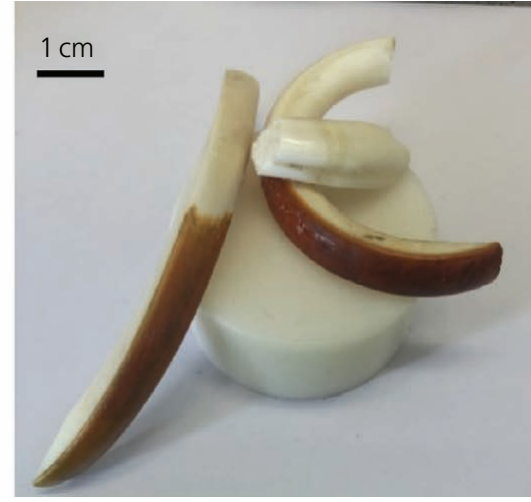

(c)

Figure 1. (a) SEM image of an incisor of the bat P. pipistrellus and images of incisors of (b) the mole rat $F$. kafuensis and (c) the beaver C. fiber

Quanta 400 FEG instrument after sputtering with gold and palladium (80:20). Fragments of a tooth of $C$. fiber were also analyzed by wavelength-dispersive X-ray spectroscopy (WDS) with a Zeiss Gemini 300 scanning electron microscope, equipped with an Oxford Wave 500 detector.

For X-ray powder diffraction (XRD), the samples were ground to a fine powder as follows: the teeth were transversally cut with a Proxxon FBS 230/E fine drilling and polishing tool equipped with a diamond-coated cutting disk. Fine powders of enamel or dentin (a few milligrams per sample), were obtained from corresponding areas of the cut teeth with the same instrument using a diamondcoated drill. The obtained powder was used for XRD with a Bruker D8 Advance diffractometer (copper $(\mathrm{Cu}) \mathrm{K} \alpha$ radiation, $\lambda=1.54 \AA$ ) on a silicon single-crystal-sample holder to minimize background scattering. For the qualitative phase analysis with the Bruker software Diffrac.Suite Eva V1, the pattern of hydroxyapatite from the International Centre for Diffraction Data (ICDD) database (number 09-0432) was used as reference. Rietveld refinement for the calculation of the lattice parameters and the crystallite sizes was performed with the Bruker software Topas 5.0. For Rietveld refinement, the instrumental correction was included as determined with a standard powder sample of lanthanum hexaboride $\left(\mathrm{LaB}_{6}\right)$ (from the National Institute of Standards and Technology, standard reference material SRM 660b). The size of the crystallites was calculated by Rietveld refinement, based on the Scherrer equation after correction for instrumental peak broadening. ${ }^{19}$

\section{Results and discussion}

The investigated samples are shown in Figure 1. The incisors of P. pipistrellus had a size of about $1 \mathrm{~mm}$, those of $F$. kafuensis had a curved length of about 3-4 cm and those of $C$. fiber had a curved length of about $8 \mathrm{~cm}$. All teeth were white, except for those of $C$. fiber, which had a distinct reddish-brown color on the convex part, representing the fully mature enamel surface of the distal part, including the cutting edge.
Figure 2 shows SEM images of the cross-section of a human deciduous tooth (Figure 2(a)). Starting from the DEJ, the hydroxyapatite prisms are arranged perpendicular to the outer enamel surface (Figure 2(b)). ${ }^{20}$ Within the prismatic enamel, the crystallites are oriented with their long axes in a similar fashion (Figure 2(c), arrows). The dentin consists of rather disordered crystallites, embedded in an organic matrix that is pervaded by parallel dentin tubuli with a diameter of about $1 \mu \mathrm{m}$ (Figure 2(d), arrows).

Figure 3 shows SEM images of a fractured tooth of $C$. fiber. The surface of the tooth is covered with a brown iron-rich layer on the highly oriented enamel crystals below (Figure 3(a)). The entire
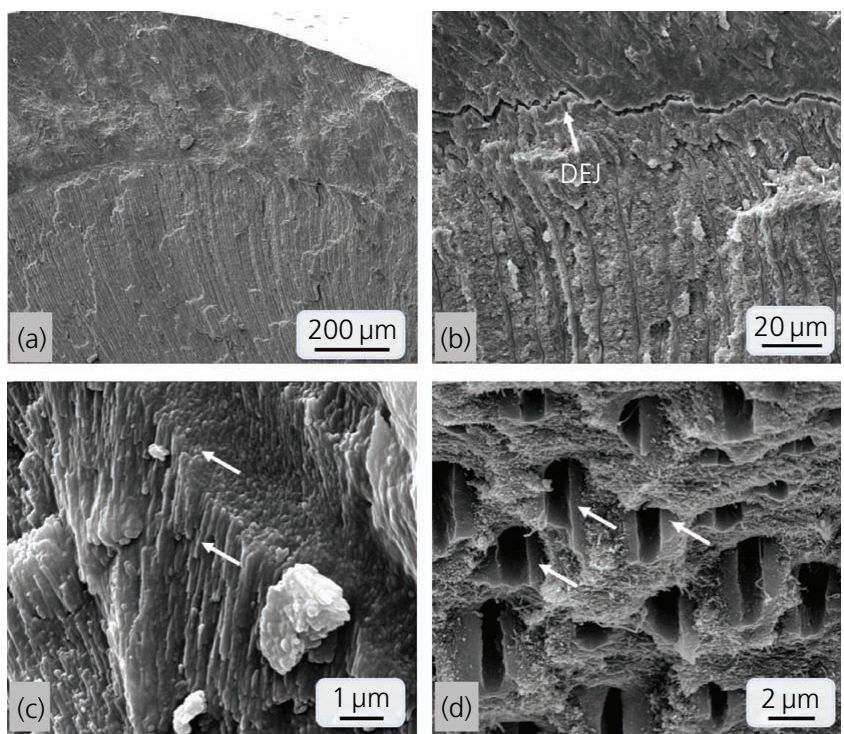

Figure 2. SEM images of a human deciduous tooth: (a) overview; (b) magnification of the DEJ; (c) magnification of the enamel; (d) magnification of the dentin 
Bioinspired, Biomimetic and Nanobiomaterials Volume 8 Issue BBN4
Optimized biological tools: ultrastructure of rodent and bat teeth compared to

human teeth

Luebke, Loza, Prymak et al.
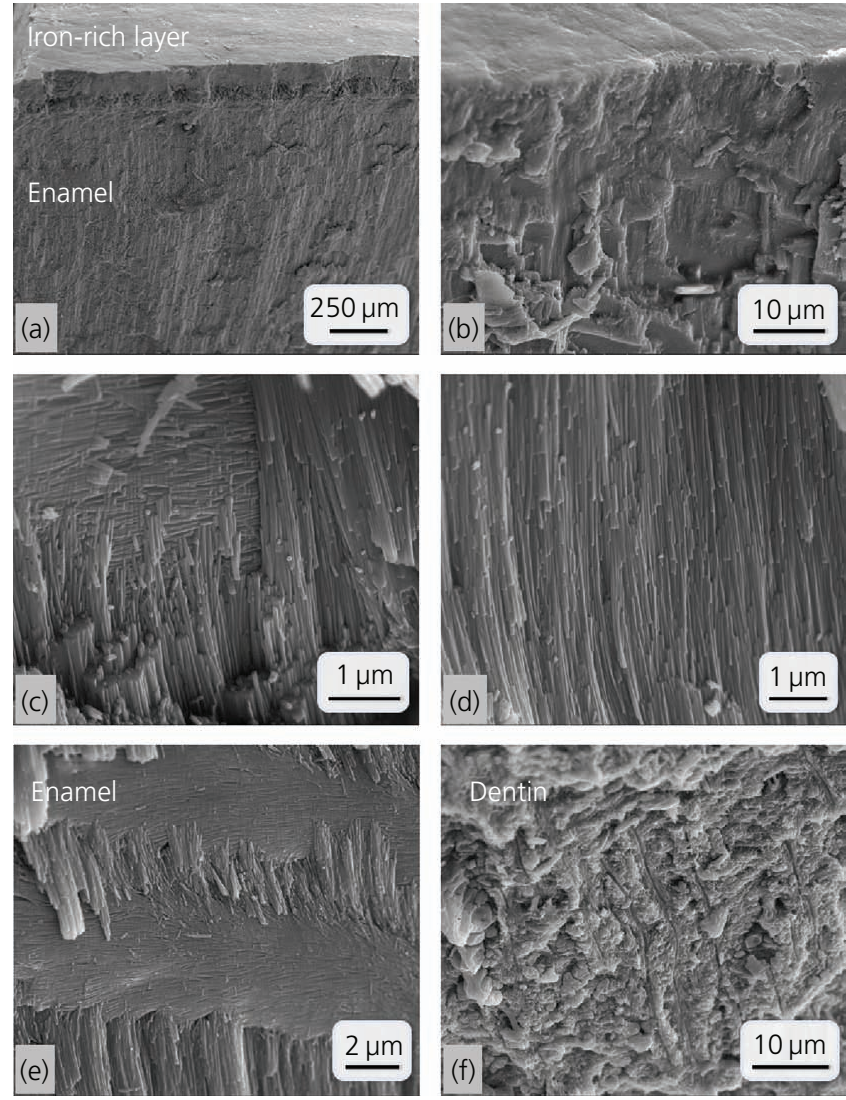

Figure 3. SEM images of a tooth of $C$. fiber: $(a, b)$ cross-sections of the tooth surface and the enamel, showing the iron-rich enamel part (convex) and the white enamel part (concave), respectively; (c-e) the enamel at higher magnification; ( $f$ ) the dentin microstructure

enamel layer is $\sim 1 \mathrm{~mm}$ thick. In the cross-section, the first $\sim 200 \mu \mathrm{m}$ thick outermost layer below the surface appears distinctly separated from the underlying bulk of the enamel, indicating a structural difference (Figure 3(a)). At higher magnification, this layer consists of parallel crystallites that form flat layers oriented perpendicularly to the tooth surface (Figure 3(b)). The EDX analysis (Figure 4) of the enamel confirms the presence of iron in the outer part of this layer besides the major phase calcium phosphate. The uncolored enamel consists of long prisms that either form an interwoven pattern close to the surface with angles of about $90^{\circ}$ between them (Figure $3(\mathrm{c})$ ) or proceed in a very regular parallel arrangement (close to the DEJ) (Figure 3(d)). Their thickness is relatively uniform and amounts to $30-50 \mathrm{~nm}$. The dentin resembles that of human teeth as it is being pervaded with long tubuli with similar diameters. The diameter of the tubuli is $2-3 \mu \mathrm{m}$.

Figure 5 shows a line scan across the iron-rich part of the $C$. fiber tooth recorded by WDS for the elements iron, calcium (Ca), phosphorus and oxygen, including an SEM image of the sampled region. The thickness of the iron-rich layer is estimated to be around

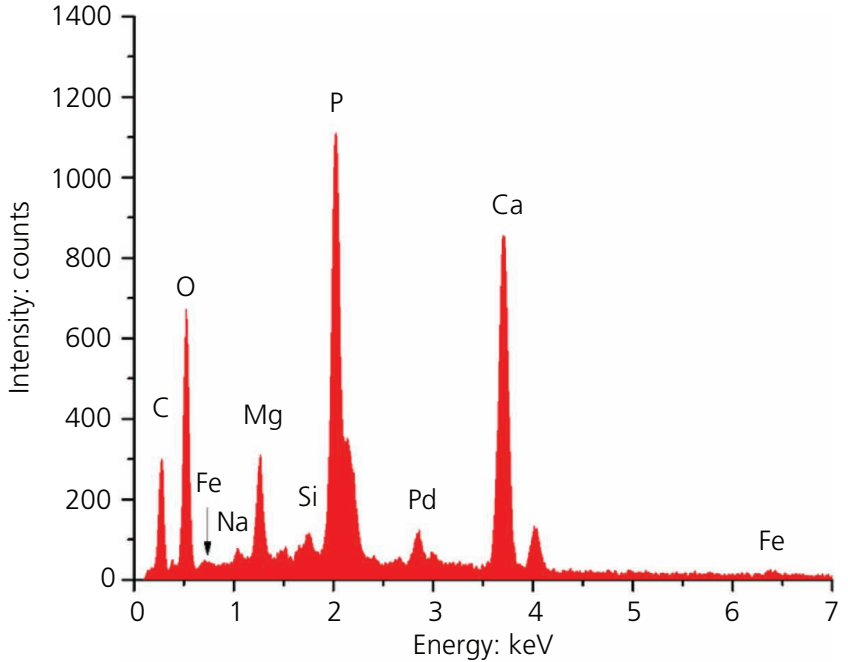

(a)

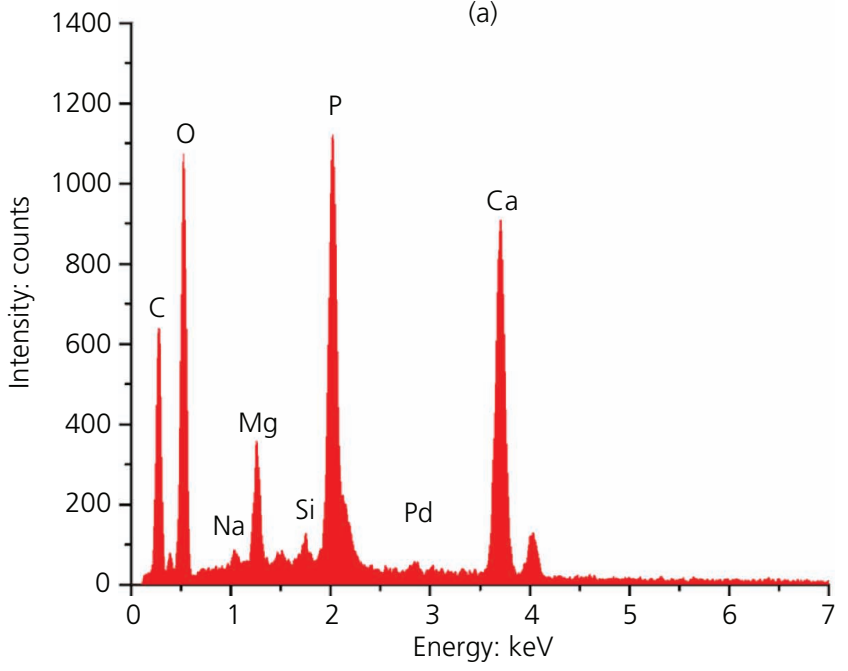

(b)

Figure 4. EDX analysis of the enamel layer of an incisor of $C$. fiber close to the cutting edge: (a) iron-rich enamel and (b) white enamel. Carbon $(\mathrm{C})$, oxygen $(\mathrm{O})$, iron $(\mathrm{Fe})$, magnesium $(\mathrm{Mg})$, phosphorus $(\mathrm{P})$ and calcium (Ca) signals originate from the tooth. The silicon ( $\mathrm{Si}$ ) and palladium $(\mathrm{Pd})$ signals are caused by the sample holder and sputtering, respectively. The tooth mineral consists of calcium phosphate. Due to the irregular nature of the samples, EDX did not give quantitative results. However, the calcium-phosphorus molar ratio was around 1·5:1, as expected for a biological apatite

$10 \mu \mathrm{m}$. Iron is located only in the outermost layer of the enamel, which otherwise consists of calcium phosphate. Earlier, Gordon et $a l^{21}$ had identified the mineral in the brown iron-containing layer in C. fiber as a mixture of ferrihydrite, $\mathrm{Fe}_{2} \mathrm{O}_{3} \cdot x \mathrm{H}_{2} \mathrm{O}$, and amorphous iron-calcium phosphate. It was not possible to record an EDX map due to the lower sensitivity of EDX compared to WDS.

SEM images of a tooth of F. kafuensis are shown in Figure 6. The enamel is $\sim 250 \mu \mathrm{m}$ thick, in good agreement with the result of Teleky et al., ${ }^{22}$ with a uniform structure. The enamel consists of alternating layers of crystallite prisms tilted against each other at 
Bioinspired, Biomimetic and Nanobiomaterials Volume 8 Issue BBN4
Optimized biological tools: ultrastructure of rodent and bat teeth compared to

human teeth

Luebke, Loza, Prymak et al.

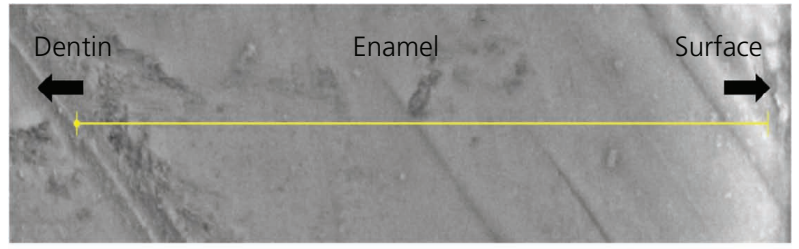

(a)
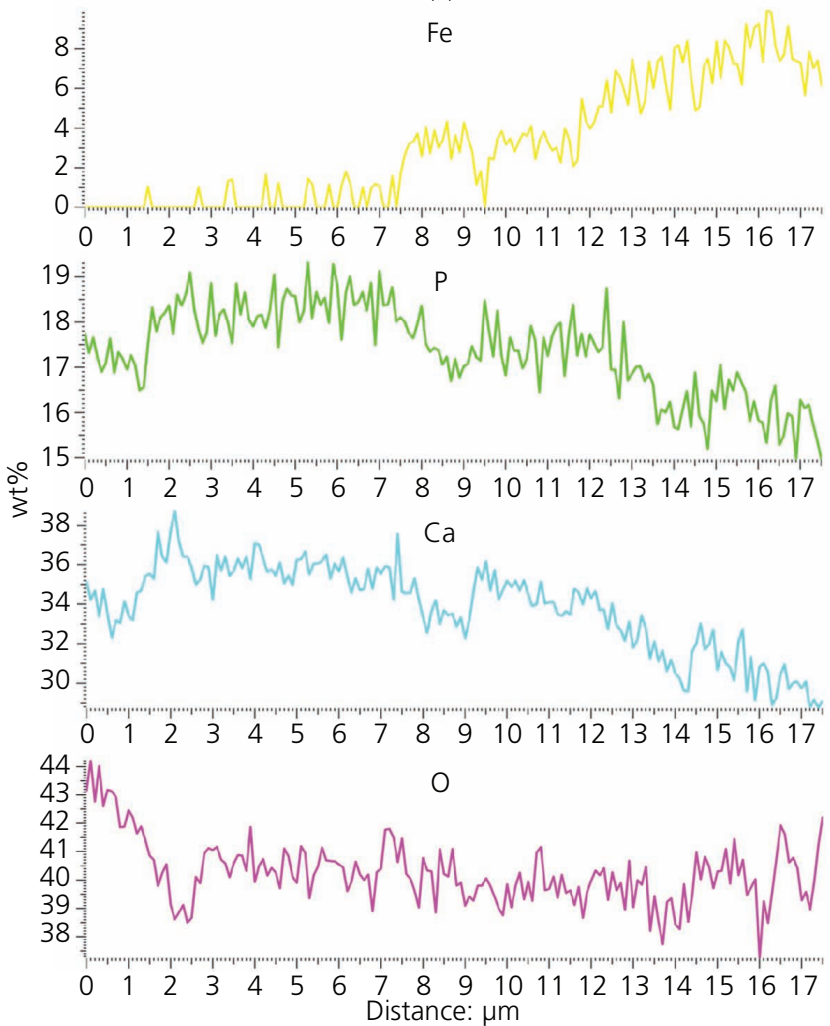

(b)

Figure 5. (a) SEM image and (b) WDS line scans of iron, phosphorus, calcium and oxygen across an incisor of $C$. fiber with a brown surface (outer convex part)

an angle of less than $90^{\circ}$. The prisms are equally densely arranged as in the tooth of $C$. fiber. The dentin appears less organized and contains thin and long tubuli with a thickness of less than $1 \mu \mathrm{m}$. EDX mapping of the tooth cross-section (Figure 7) shows the calcium phosphate biomineral with a stronger calcium signal in enamel compared to dentin, indicating an increasing degree of mineralization from dentin to enamel. The molar ratio of calcium to phosphorus was about $1 \cdot 46: 1$ across the scan, indicating the presence of calcium-deficient biological apatite. ${ }^{5,9}$

Figure 8 shows a representative XRD pattern of the dentin of F. kafuensis. The mineral phase consists of hydroxyapatite, with a crystallite size of $9 \pm 1 \mathrm{~nm}$ and lattice parameters of $9.47 \pm$ $0 \cdot 01 \AA$ ( $a$ axis) and $6 \cdot 88 \pm 0.01 \AA(c$ axis $)$, as determined by Rietveld refinement. The increased lattice parameters $a$ compared to reference hydroxyapatite ( $a=9.42 \AA$ from ICDD number 09-0432) point to an atomic substitution in the apatite structure.
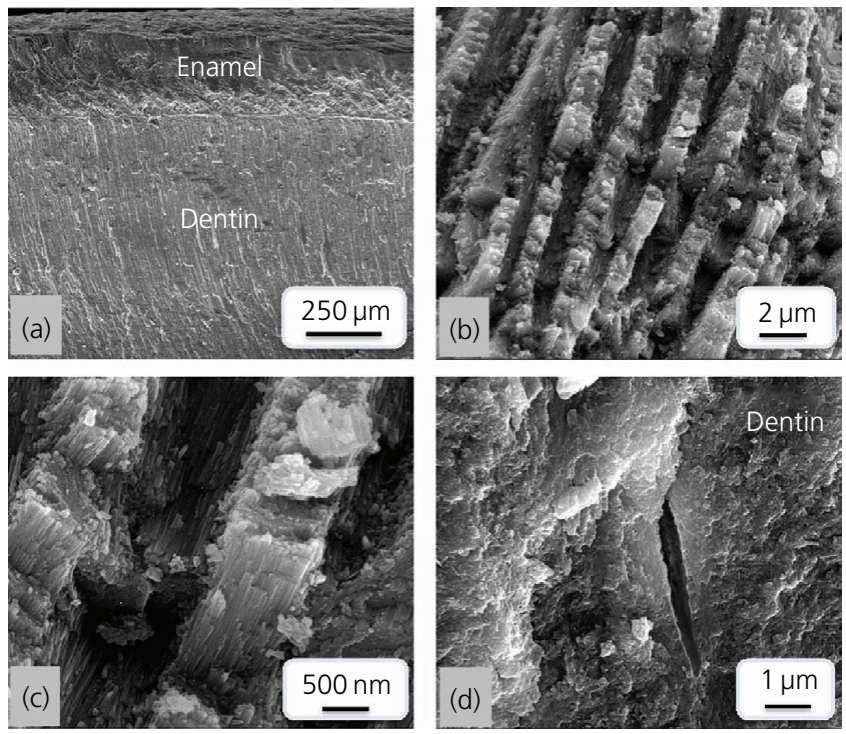

Figure 6. SEM images of a tooth of $F$. kafuensis: (a) overview with enamel and dentin; ( $b, c)$ enamel; $(d)$ dentin
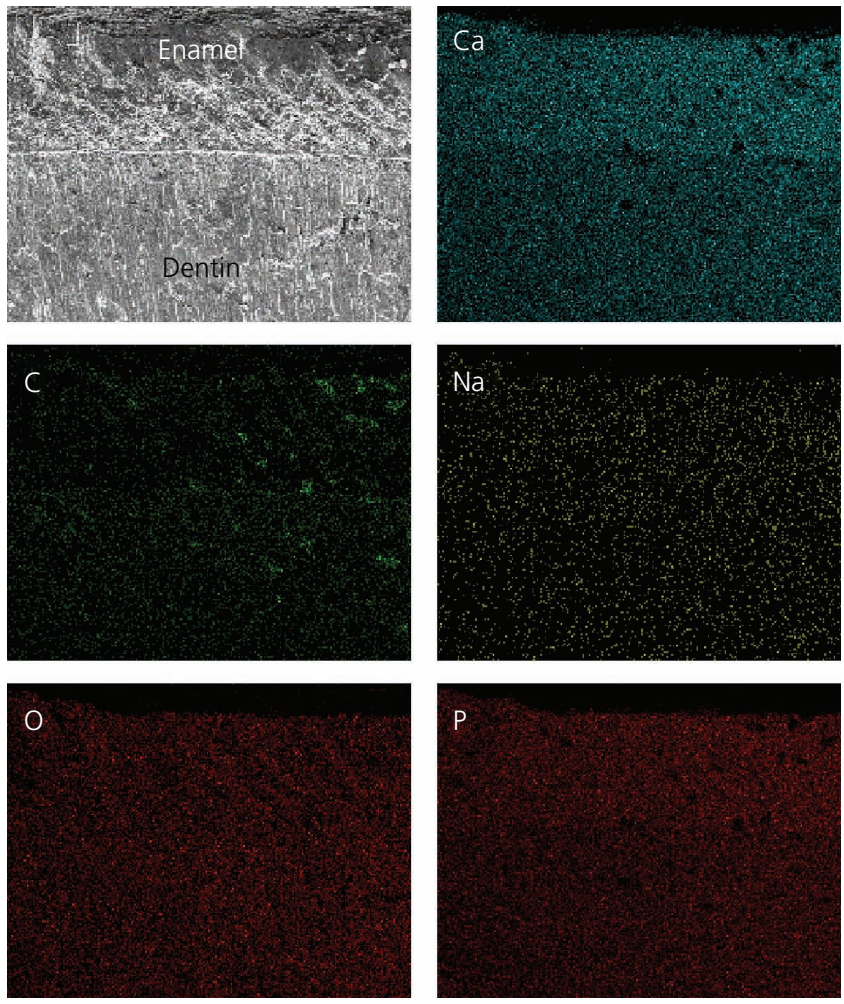

Figure 7. EDX mapping of a tooth of $F$. kafuensis, showing the distribution of the elements across the tooth (enamel and dentin). The enamel is higher mineralized (calcium, phosphorus) and contains less organic matrix (carbon) 
Bioinspired, Biomimetic and Nanobiomaterials Volume 8 Issue BBN4
Optimized biological tools: ultrastructure of rodent and bat teeth compared to

human teeth

Luebke, Loza, Prymak et al.

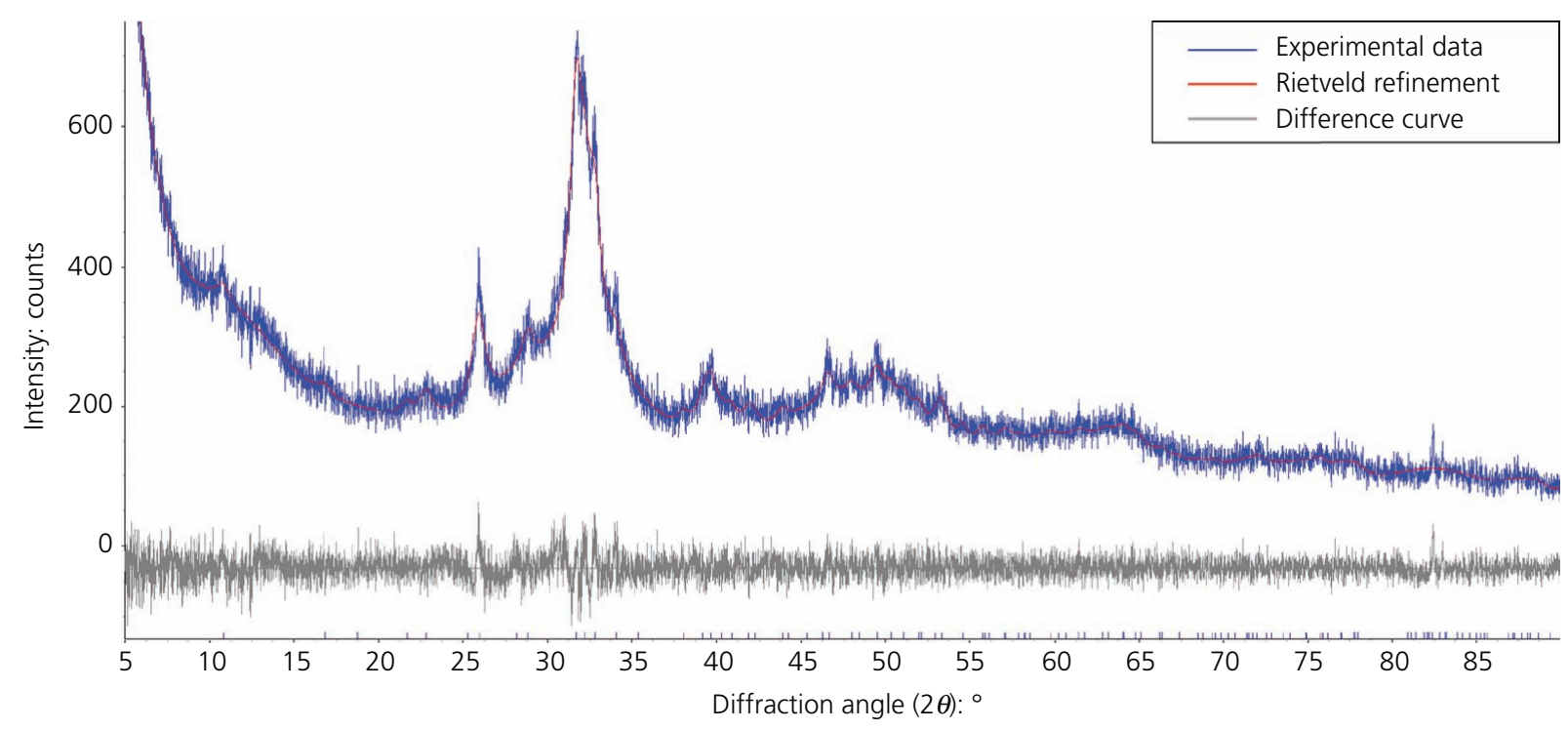

Figure 8. Representative $X$-ray powder diffractogram of $F$. kafuensis dentin with a fit to hydroxyapatite as the only crystalline phase by Rietveld refinement $\left(R_{\mathrm{wp}}=7 \cdot 2\right)$

It was not possible to analyze the enamel of $F$. kafuensis teeth by XRD because not enough material could be obtained from the very thin enamel layer.

Figure 9 shows SEM images of a tooth of $P$. pipistrellus. The tooth is very thin with a large pulpa cavity. Interestingly, the enamel takes up almost one-third of the thickness, while the rest is dentin (Figure 9(a)). Compared to the other species, the
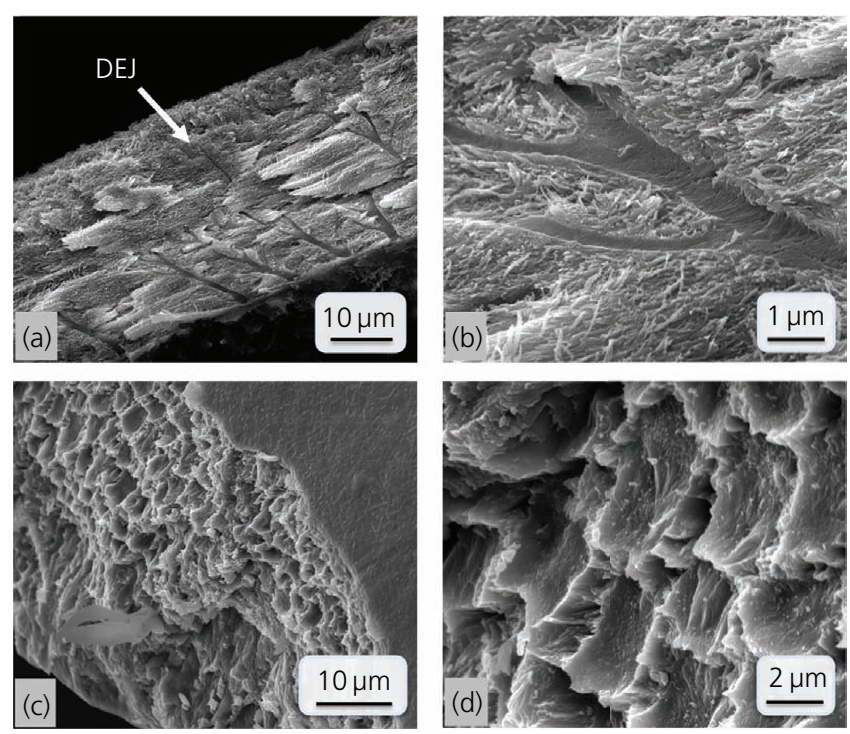

Figure 9. SEM images of the fracture surface of a tooth of P. pipistrellus: (a) overview (enamel: top; dentin: bottom); (b) dentin layer; (c, d) enamel layer enamel-dentin ratio is much larger and thus the tooth can be expected to be harder than the three other examples, which is in good agreement with its function as a sharp piercing tool. Although the dentin is very thin, the tubuli have the same basic dimensions as in human teeth, but contain more ramifications close to the DEJ (Figure 9(b)). A higher magnification of the enamel shows needle-shaped prisms that are oriented parallel to each other and to the tooth surface (Figure 9(c)).

The long axes of the prisms are oriented longitudinally from the base of the tooth toward the sharp tip. Such an arrangement will increase the stability of the tooth in the direction where the bat stabs its prey, which consists of insects whose exoskeletons can be quite hard and tough. This arrangement differs from human and rodent enamel and is reminiscent of the structural organization of shark teeth, which have a similar function. ${ }^{23}$ The prisms are very densely distributed with very little interprismatic enamel (Figure 9(d)). Figure 10 shows that the mineral phase consists exclusively of calcium phosphate. XRD was not possible due to the very small amount of material in the bat teeth.

Human enamel has a highly regulated structure. The needle-like hydroxyapatite crystallites, which are roughly rectangular in cross-section, ${ }^{24}$ form bundles that are held together by organic molecules into larger structures, called enamel prisms. These prisms are arranged perpendicular to the DEJ toward the outer enamel surface. They can be twisted together or change their direction in some areas in order to increase the hardness of the overall structure. ${ }^{25}$ The hardness of human enamel is $3.5 \pm$ $0.5 \mathrm{GPa}$, and the reduced elastic modulus is $77 \pm 10 \mathrm{GPa}^{26}$ Human third molar dentin shows a hardness of $1 \cdot 0 \pm 0 \cdot 15 \mathrm{GPa}$ and a reduced elastic modulus of $23 \cdot 7 \pm 2 \cdot 6 \mathrm{GPa}^{26}$ The structure 
Optimized biological tools: ultrastructure of rodent and bat teeth compared to

human teeth

Luebke, Loza, Prymak et al.

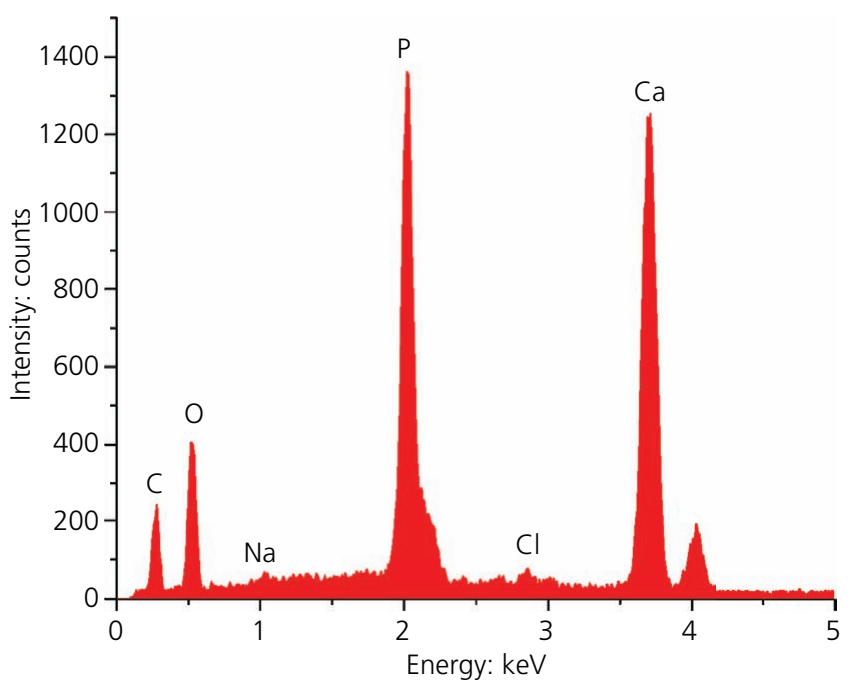

Figure 10. EDX-measurement of a tooth of $P$. pipistrellus, showing the presence of calcium phosphate mineral. Due to the irregular nature of the sample, EDX did not give quantitative results. However, the calcium-phosphorus molar ratio was around $1 \cdot 5: 1$, as expected for biological apatite

of human molar teeth arises from the diet: their main functions are to chew, to tear and to cut.

While humans can be considered as omnivorous (and hence chew food of variable hardness), echolocating bats such as Pipistrellus use their incisors primarily to catch and kill their insect prey. ${ }^{27}$ In contrast, incisors of the two rodent species in this study are confronted with more demanding tasks: besides gnawing all kinds of plant material for food, beavers use their incisors extensively to cut trees for building dams, lodges and canals ${ }^{28-30}$ and African mole rats, including Fukomys, dig extensive burrow systems in soils of varying hardness with their incisors. ${ }^{31-33}$ In contrast to many other mammals, most rodents show monophyodonty - that is, they do not shed their primary teeth.

Rodent incisors are characterized by a sophisticated structure. The enamel is present in only one small, lamellar film on the outer part of the tooth. Furthermore, as an adaptation to the requirements described earlier, rodent incisors grow continuously and their length is controlled by continuous abrasion caused by their comparably hard and fibrous diet - for example, wood, nuts or other plant materials - or even digging through soils like in the case of Fukomys. ${ }^{32,33}$ Their particular structure with an enamel layer located on the labial (outer) surface is supported only by the roughly cylindrical rest of the tooth that consists of dentin. This is responsible for the rodents' ability to maintain a sharp cutting edge always. During chewing, the softer inner dentin layer is eroded by the outer, harder enamel, leaving a sharp edge both on the upper and lower jaw incisors. Beaver enamel can be divided into three enamel types: the pigmented iron-containing outer enamel, the underlying non-pigmented enamel and the inner enamel. The hardness decreases from the outside to the inside: 5-6 GPa for the pigmented iron-containing enamel and 4-5 GPa for the non-pigmented white enamel. ${ }^{21}$ The hardness correlates with the function of the teeth and the diet of the vertebrate. ${ }^{30}$

\section{Conclusions}

Teeth of two rodent species, namely the European beaver $C$. fiber and the African mole rat $F$. kafuensis, and of the bat P. pipistrellus all consist of calcium phosphate with a high similarity to human teeth. A highly mineralized outer enamel layer surrounds the less mineralized inner dentin. In comparison to human teeth, the enamel layer in the two rodents and in the bat is much thinner. The dentin tubuli in the three species have a diameter (between 1 and $3 \mu \mathrm{m}$ ) comparable to that of human teeth, making them relatively large in the small bat teeth. The beaver teeth contain a thin layer $(\sim 10 \mu \mathrm{m})$ of iron-containing mineral (most likely iron oxide) on their outer surface, probably to increase their hardness.

\section{Acknowledgements}

The authors thank Mrs Carola Fischer for help with the sample preparation. The authors thank Jakob Epple for the donation of a deciduous tooth. This research did not receive any specific grant from funding agencies in the public, commercial or not-forprofit sectors.

\section{REFERENCES}

1. Dunlop JWC and FratzI P (2010) Biological composites. Annual Review of Materials Research 40(1): 1-24.

2. Fratzl P and Weinkamer R (2007) Nature's hierarchical materials. Progress in Materials Science 52(8): 1263-1334.

3. Reznikov N, Shahar R and Weiner S (2014) Bone hierarchical structure in three dimensions. Acta Biomaterialia 10(9): 3815-3826.

4. Lowenstam HA and Weiner S (1989) On Biomineralization. Oxford University Press, New York, NY, USA.

5. LeGeros RZ (1981) Apatites in biological systems. Progress in Crystal Growth and Characterization 4(1-2): 1-45.

6. Baeuerlein E, Behrens P and Epple M (2007) Handbook of Biomineralization. Wiley-VCH, Weinheim, Germany.

7. Mann S (2001) Biomineralization. Oxford University Press, Oxford, UK.

8. Teaford MF, Smith MM and Ferguson MWJ (2000) Development, Function and Evolution of Teeth. Cambridge University Press, Cambridge, UK.

9. Dorozhkin SV and Epple M (2002) Biological and medical significance of calcium phosphates. Angewandte Chemie International Edition 41(17): 3130-3146.

10. Sander PM (2000) Prismless enamel in amniotes: terminology, function, and evolution. In Development, Function and Evolution of Teeth (Teaford MF, Smith MM and Ferguson MWJ (eds)). Cambridge University Press, Cambridge, UK, pp. 92-106.

11. Koenigswald W (2004) The three basic types of schmelzmuster in fossil and extant rodent molars and their distribution among rodent clades. Palaeontographica Abteilung A - Stuttgart 270(4-6): 95-132.

12. Busch S, Schwarz U and Kniep R (2001) Morphogenesis and structure of human teeth in relation to biomimetically grown fluoroapatite-gelatine composites. Chemistry of Materials 13(10): 3260-3271.

13. Ang SF, Bortel EL, Swain MV, Klocke A and Schneider GA (2010) Size-dependent elastic/inelastic behavior of enamel over millimeter and nanometer length scales. Biomaterials 31(7): 1955-1963. 
Bioinspired, Biomimetic and Nanobiomaterials Volume 8 Issue BBN4
Optimized biological tools: ultrastructure of rodent and bat teeth compared to human teeth Luebke, Loza, Prymak et al.
14. Jeng YR, Lin TT, Hsu HM, Chang HJ and Shieh DB (2011) Human enamel rod presents anisotropic nanotribological properties. Journal of the Mechanical Behavior of Biomedical Materials 4(4): 515-522.

15. Imbeni V, Kruzic JJ, Marshall GW, Marshall SJ and Ritchie RO (2005) The dentin-enamel junction and the fracture of human teeth. Nature Materials 4(3): 229-232.

16. Jambura PL, Pfaff C, Underwood CJ, Ward DJ and Kriwet J (2018) Tooth mineralization and histology patterns in extinct and extant snaggletooth sharks, Hemipristis (Carcharhiniformes, Hemigaleidae) evolutionary significance or ecological adaptation? PLOS ONE 13(8): e0200951.

17. Weiner S and Addadi L (1997) Design strategies in mineralized biological materials. Journal of Materials Chemistry 7(5): 689-702.

18. Fabritius $H$, Sachs $C$, Triguero $P$ and Raabe D (2009) Influence of structural principles on the mechanics of a biological fiber-based composite material with hierarchical organization: the exoskeleton of the lobster Homarus americanus. Advanced Materials 21(4): 391-400.

19. Klug HP and Alexander LE (1974) X-ray Diffraction Procedures for Polycrystalline and Amorphous Materials. Wiley-Interscience, New York, NY, USA.

20. He LH and Swain MV (2008) Understanding the mechanical behaviour of human enamel from its structural and compositional characteristics. Journal of the Mechanical Behavior of Biomedical Materials 1(1): 18-29.

21. Gordon LM, Cohen MJ, MacRenaris KW et al. (2015) Amorphous intergranular phases control the properties of rodent tooth enamel. Science 347(6223): 746-750.

22. Teleky J, Melnik O, Toth T et al. (2018) Craniometry of the Slovak northeastern beavers (Castor fiber) in comparison with the Ukrainian and Polish populations and contribution to the knowledge of the enamel thickness of beaver's incisors. Biologia 73(4): 379-387.
23. Enax J, Janus AM, Raabe D, Epple M and Fabritius HO (2014) Ultrastructural organization and micromechanical properties of shark tooth enameloid. Acta Biomaterialia 10(9): 3959-3968.

24. Frazier PD (1968) Adult human enamel: an electron microscopic study of crystallite size and morphology. Journal of Ultrastructure Research 22(1): 1-11.

25. Macho GA, Jiang $Y$ and Spears IR (2003) Enamel microstructure - a truly three-dimensional structure. Journal of Human Evolution 45(1): $81-90$.

26. Habelitz S, Marshall GW, Balooch M and Marshall SJ (2002) Nanoindentation and storage of teeth. Journal of Biomechanics 35(7): 995-998.

27. Self CJ (2015) Dental root size in bats with diets of different hardness. Journal of Morphology 276(9): 1065-1074.

28. Nowak RM (1991) Walker's Mammals of the World. John Hopkins University Press, Baltimore, MD, USA, vol. 1, pp. 364-367.

29. Stefen C (2009) Intraspecific variability of beaver teeth (Castoridae: Rodentia). Zoological Journal of the Linnean Society 155(4): 926-936.

30. Stefen C, Habersetzer J and Witzel U (2016) Biomechanical aspects of incisor action of beavers (Castor fiber L.). Journal of Mammalogy 97(2): 619-630.

31. Burda H (2003) Adaptations for subterranean life. In Grzimek's Animal Life Encyclopedia: Mammals I (Kleiman DG, Geist V, Hutchins M and McDade MC (eds)). Gale Group, Farmington Hills, MI, USA, vol. 12, pp. 69-78.

32. Van Daele P, Herrel A and Adriaens D (2009) Biting performance in teeth-digging African mole-rats (Fukomys, Bathyergidae, Rodentia). Physiological and Biochemical Zoology 82(1): 40-50.

33. Rodrigues HG, Hautier L and Evans AR (2017) Convergent traits in mammals associated with divergent behaviors: the case of the continuous dental replacement in rock-wallabies and African molerats. Journal of Mammalian Evolution 24(3): 261-274.

\section{How can you contribute?}

To discuss this paper, please submit up to 500 words to the journal office at journals@ice.org.uk. Your contribution will be forwarded to the author(s) for a reply and, if considered appropriate by the editor-in-chief, it will be published as a discussion in a future issue of the journal.

ICE Science journals rely entirely on contributions from the field of materials science and engineering. Information about how to submit your paper online is available at www.icevirtuallibrary.com/page/authors, where you will also find detailed author guidelines. 